\title{
ACOUSTICAL TRANSMISSION CHANNEL OF THE INFORMATION \\ IN UNDERGROUND TURNPIKE WATER SUPPLY SYSTEMS
}

\author{
V. Zibrov ${ }^{1}$, D. Maltseva ${ }^{2}$ \\ DOI: http://doi.org/10.15350/L_26/8/22
}

\section{Abstract}

In the given operation agency of the reflected radial waves on interfaces of the several medium having different density, on the "plane» wave which is passed round in the underground turnpike water pipeline is observed.

Keywords: an acoustical wave, the turnpike water pipeline, reflexions, reverberation, modes.

The field of examinations of an acoustical transmission channel of the information (ATCI) in the closed underground water pipeline is feebly studied now. Unlike the acoustical communications applied in open water medium where impairment is a principal cause of decrease of a signal, acoustical applied reverberation in the water pipeline is the most important factor of signal alteration. The limited space of the water pipeline creates a serious multiple-beam advance of waves. For acoustical radiation of long-range act in open water medium of theoretical and scientific bottoms it is usually enough for exposition ATCI, but for modeling ATCI in the water pipeline the complicated methods are required applications enough. Whereas various parameters characterizes the water pipeline: pressure, temperature, viscosity and a conductivity of water, mechanical impurities and air bells in water, a material, diameter of the water tube, etc. Gives the warrant to guess, that these parameters will call certain difficulties at organization ATCI $[1,2,3]$.

Paper purpose is definition of agency of the mirrored radial waves on interfaces of the several medium having different density, on extending by a «plane» wave.

The underground water tube is subject to act of radial loads of squeezing and the circle loads of detrusion calling strain of a pipe and short-term decrease of diameter. We will observe a case when the underground pipe is not elastic (an acoustical wave it is passed round, both in a pipe, and through pipe walls in a ground), and also it is not subject to strains and modifications of diameter (fig. 1).

${ }^{1}$ Valery Zibrov, Candidate of Technical Sciences, Associate Professor, Don State Technical University, Russia.

2Dzhamilya Maltseva, graduate student, Don State Technical University, Russia. 


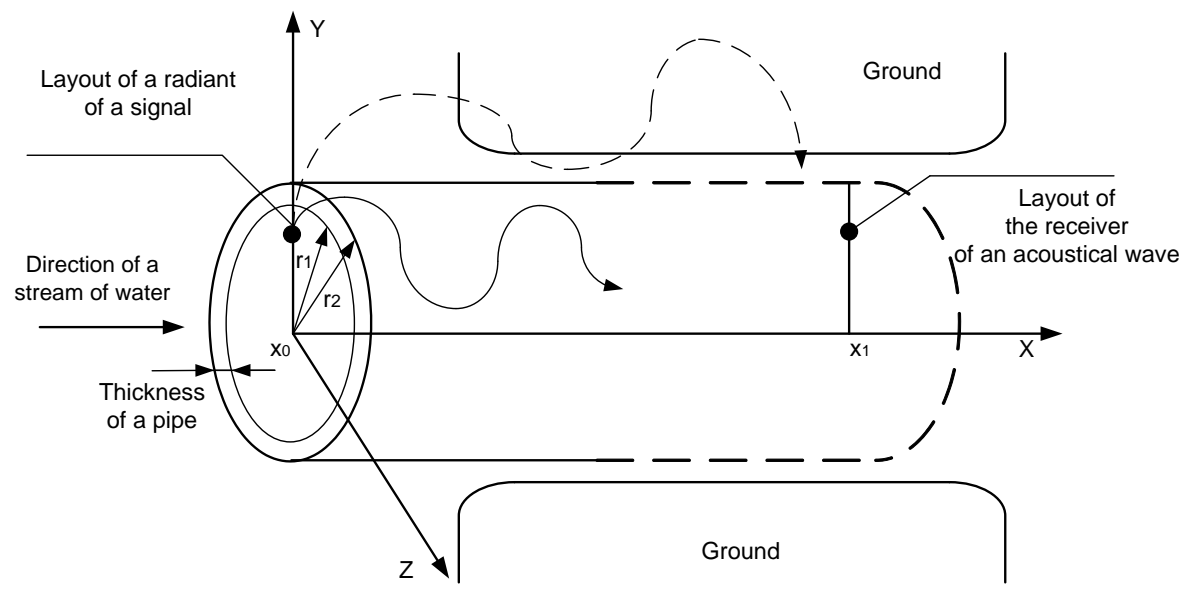

Fig. 1. Extending of an acoustical wave to an underground pipe

The transfer function linking state variables of acoustical pressure $p_{r}$ in the radial direction and a radial velocity $v_{r}$ in interior and exterior boundary lines of a pipe $\left(r=r_{1}\right.$ and $\left.r=r_{2}\right)$ looks like: $\left[\begin{array}{c}p_{r_{2}} \\ v_{r_{2}}\end{array}\right]=\left[\begin{array}{ll}L_{1} & L_{2} \\ L_{3} & L_{4}\end{array}\right]\left[\begin{array}{c}p_{r_{1}} \\ v_{r_{1}}\end{array}\right]$, where $L_{1}=\left.\frac{p_{r_{2}}}{p_{r_{1}}}\right|_{v_{r_{1}}=0}, L_{2}=\left.\frac{p_{r_{2}}}{v_{r_{1}}}\right|_{p_{r_{1}}=0}, L_{3}=\left.\frac{v_{r_{2}}}{p_{r_{1}}}\right|_{v_{r_{1}}=0}, L_{4}=\left.\frac{v_{r_{2}}}{v_{r_{1}}}\right|_{p_{r_{1}}=0}$ - coefficients; $r_{1}$ and $r_{2}$ - interior and exterior radiuses of a pipe (fig. 2).

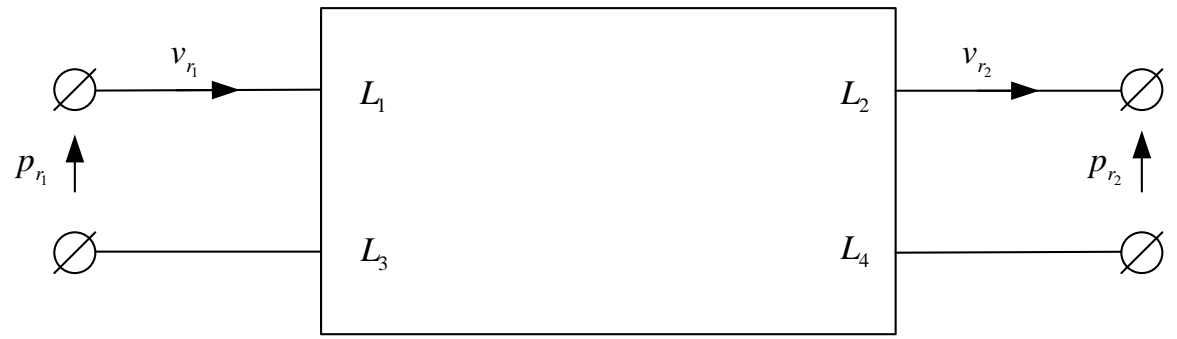

Fig. 2. Model of a pipe in the form of the four-terminal network

Expressions of acoustical pressure in the radial direction and the radial undular velocity look like $[4,5]$ :

$$
\begin{gathered}
p_{r}(\eta, \omega, r)=-i \omega \rho\left[a H_{0}^{1}(\eta r)+b H_{0}^{2}(\eta r)\right] \\
v_{r}(\eta, \omega, r)=\frac{i \eta}{\omega \rho}\left[a H_{0}^{1}(\eta r)+b H_{0}^{2}(\eta r)\right],
\end{gathered}
$$


where $H_{0}^{1}(\eta r)$ - function Hankel of the first sort, for the waves which are passing round in a direction of a ground; $H_{0}^{2}(\eta r)$ - function Hankel of the second sort, for the reflected waves which are passing round in an internal area of a pipe of a ground; $a, b$ - constants.

$$
\begin{aligned}
& \text { In the matrix form: }\left[\begin{array}{l}
p_{r_{1}} \\
v_{r_{1}}
\end{array}\right]=\left[M_{r_{1}}\left[\begin{array}{l}
a \\
b
\end{array}\right] ;\left[\begin{array}{l}
p_{r_{2}} \\
v_{r_{2}}
\end{array}\right]=\left[M_{r_{2}}\left[\begin{array}{l}
a \\
b
\end{array}\right]\right. \text {, }\right. \\
& \text { where }\left[M_{r_{1}}\right]=\left[\begin{array}{ll}
-i \omega \rho H_{0}^{1}\left(\eta r_{1}\right) & -i \omega \rho H_{0}^{2}\left(\eta r_{1}\right) \\
\frac{i \eta}{\omega \rho} H_{1}^{1}\left(\eta r_{1}\right) & \frac{i \eta}{\omega \rho} H_{1}^{2}\left(\eta r_{1}\right)
\end{array}\right]=\left[\begin{array}{ll}
r_{11} & r_{12} \\
r_{13} & r_{14}
\end{array}\right] \text {; } \\
& {\left[M_{r_{2}}\right]=\left[\begin{array}{ll}
-i \omega \rho H_{0}^{1}\left(\eta r_{2}\right) & -i \omega \rho H_{0}^{2}\left(\eta r_{2}\right) \\
\frac{i \eta}{\omega \rho} H_{1}^{1}\left(\eta r_{2}\right) & \frac{i \eta}{\omega \rho} H_{1}^{2}\left(\eta r_{2}\right)
\end{array}\right]=\left[\begin{array}{ll}
r_{21} & r_{22} \\
r_{23} & r_{24}
\end{array}\right] .}
\end{aligned}
$$

Values of constants $\left[\begin{array}{l}a \\ b\end{array}\right]=\left[M_{r_{2}}\right]^{-1}\left[\begin{array}{l}p_{r_{2}} \\ v_{r_{2}}\end{array}\right]=\left[M_{r_{1}}\right]^{-1}\left[\begin{array}{l}p_{r_{1}} \\ v_{r_{1}}\end{array}\right]$. We multiply parts of the gained expression by a matrix $\left[M_{r_{2}}\right]$ end $\left[M_{r_{1}}\right]$ :

$$
\left[\begin{array}{l}
p_{r_{2}} \\
v_{r_{2}}
\end{array}\right]=\left[M_{r_{2}}\right]\left[M_{r_{1}}\right]^{-1}\left[\begin{array}{l}
p_{r_{1}} \\
v_{r_{1}}
\end{array}\right] ;\left[\begin{array}{l}
p_{r_{1}} \\
v_{r_{1}}
\end{array}\right]=\left[M_{r_{1}}\right]\left[M_{r_{2}}\right]^{-1}\left[\begin{array}{l}
p_{r_{2}} \\
v_{r_{2}}
\end{array}\right] \text {. }
$$

Product $\left[M_{r_{2}}\right]\left[M_{r_{1}}\right]^{-1}$ - matrix of transfer from interior field in exterior field and accordingly $\left.\left\lfloor M_{r_{1}}\right\rfloor M_{r_{2}}\right\rfloor^{-1}$ - matrix of transfer from exterior field in an internal area.

Let's spend replacements in matrixes:

$$
\left[M_{r_{1} r_{2}}\right]=\frac{\left[\begin{array}{ll}
\left(r_{21} r_{14}-r_{22} r_{13}\right) & \left(-r_{21} r_{12}+r_{22} r_{11}\right) \\
\left(r_{23} r_{14}-r_{24} r_{13}\right) & \left(-r_{23} r_{12}+r_{24} r_{11}\right)
\end{array}\right]}{\Delta r_{1}} \text {, where }\left[M_{r_{1} r_{2}}\right\rfloor-\text { transfer matrix }
$$
in a direction from radius $r_{1}$ to $r_{2} ; \Delta r_{1}=r_{11} r_{14}-r_{12} r_{13}$.

We spend replacements in expression $\Delta r_{1}$ functions Hankel, we discover a Wronskian for functions Hankel, and further we define coefficients $L_{1}-L_{4}$ :

$$
L_{1}=-i \frac{\pi}{4} \eta r_{1}\left[H_{0}^{1}\left(\eta r_{2}\right) H_{1}^{2}\left(\eta r_{1}\right)-H_{0}^{2}\left(\eta r_{2}\right) H_{1}^{1}\left(\eta r_{1}\right)\right]
$$




$$
\begin{aligned}
L_{2} & =\frac{\pi}{4} \eta r_{1} \rho \omega\left[H_{0}^{1}\left(\eta r_{2}\right) H_{0}^{2}\left(\eta r_{1}\right)-H_{0}^{2}\left(\eta r_{2}\right) H_{0}^{1}\left(\eta r_{1}\right)\right] ; \\
L_{3} & =\frac{\pi}{4} \frac{\eta r_{1}}{\rho \omega}\left[H_{1}^{1}\left(\eta r_{2}\right) H_{1}^{2}\left(\eta r_{1}\right)-H_{1}^{2}\left(\eta r_{2}\right) H_{1}^{1}\left(\eta r_{1}\right)\right] ; \\
L_{4} & =-i \frac{\pi}{4} \eta r_{1}\left[H_{1}^{2}\left(\eta r_{2}\right) H_{0}^{1}\left(\eta r_{1}\right)-H_{1}^{1}\left(\eta r_{2}\right) H_{0}^{2}\left(\eta r_{1}\right)\right] .
\end{aligned}
$$

Thus, gained rated by coefficients $L_{1}-L_{4}$, through a transfer matrix, allow to spot magnitude of the acoustical wave which are passing round through walls of a pipe and a ground and to size up its agency on a «plane» wave.

Let's carry out calculation of acoustical pressure, by means of interactive system MatLab, in a turnpike steel water pressure tube jointless (diameter $199 \mathrm{~mm}$, a thickness $2.0 \mathrm{~mm}$ ). Frequency of an initial pulse $55 \mathrm{KHz}$; distance to the audible signal receiver $400 \mathrm{~m}$. Time fields of extending of acoustical pressure, are resulted in a figure 3 .

Acoustical velocity of extending of a wave depends on a strength of materials (water, a steel, a ground) which distribute power waves. In a figure 3, a reduced form of the pulses which have transited in a ground through a steel pipe. On a pulse shape it is visible, that in a ground the plane wave will not be passed round, therefore it is possible to guess, that the given pulse in the form of noise will be superimposed on a plane wave which is passed round in a pipe. Also on a receiver entry the faster modes which have arrived on walls of a steel pipe arrive. Other modes which are passing round through longer paths of transfer, including possible reflexions from walls of a steel pipe and the past through a ground of various density are observed also [6,7]. At decrease of frequency of an initial pulse to $5 \mathrm{KHz}$ not changing diameter of a pipe, we observe fadings of the mirrored signals which have transited through walls of a steel pipe and fields of a ground on $14 \mathrm{~dB}$.

Field of extending of acoustical pressure through a pipe in a ground

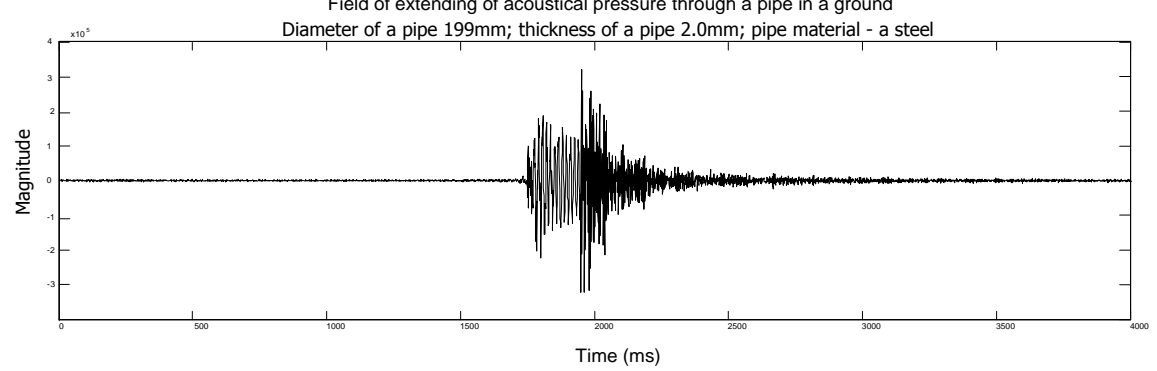




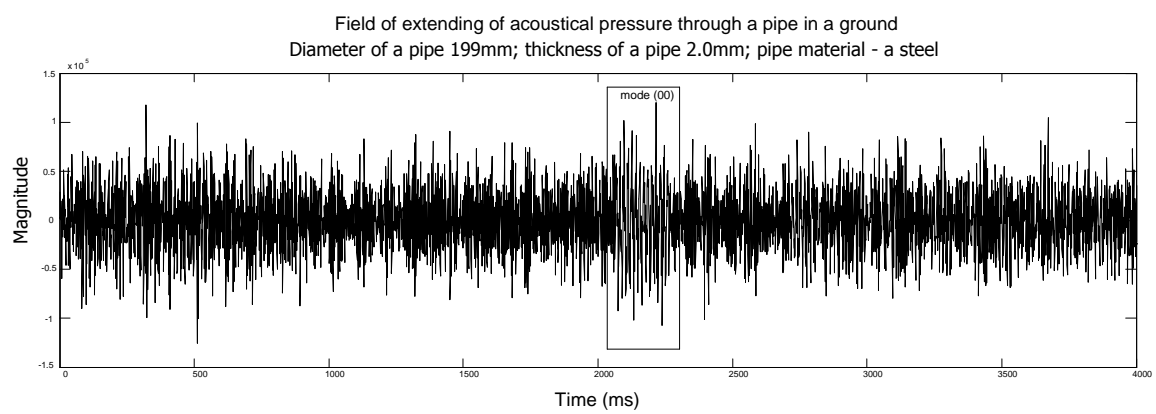

Field of extending of acoustical pressure through a pipe in a ground

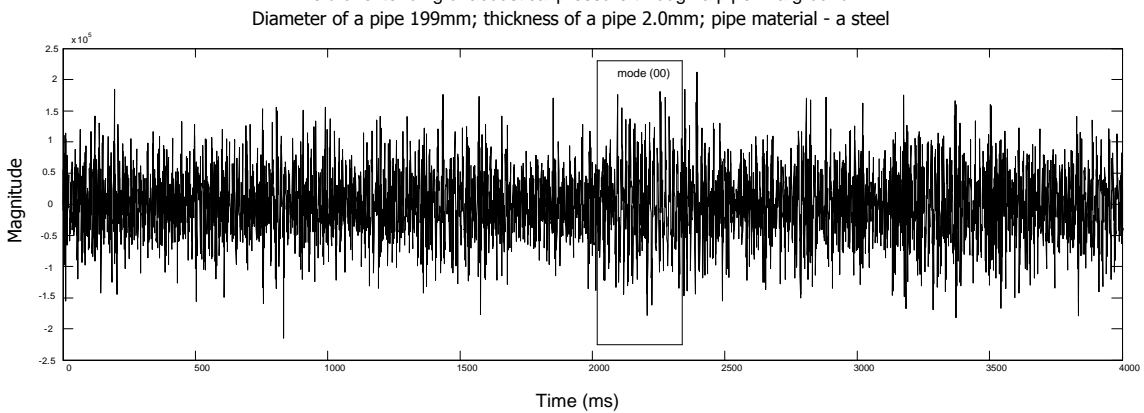

Field of extending of acoustical pressure through a pipe in a ground

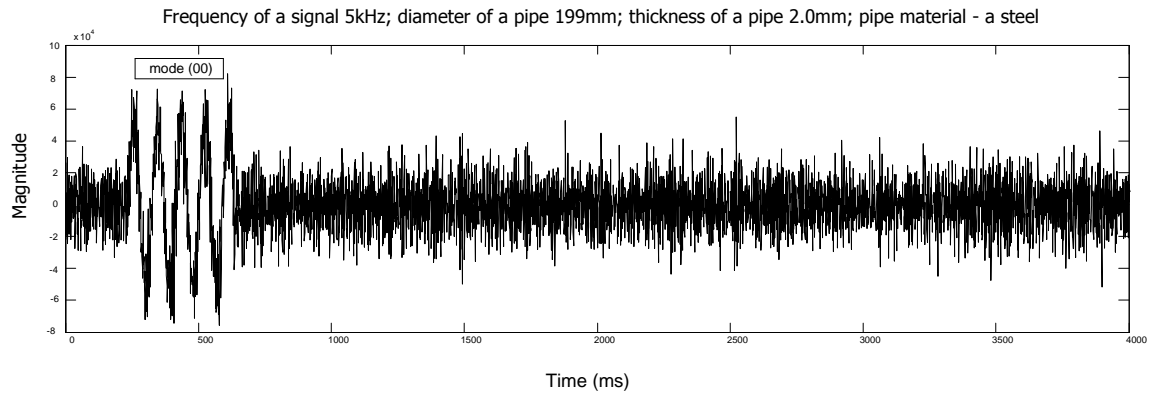

Field of extending of acoustical pressure through a pipe in a ground

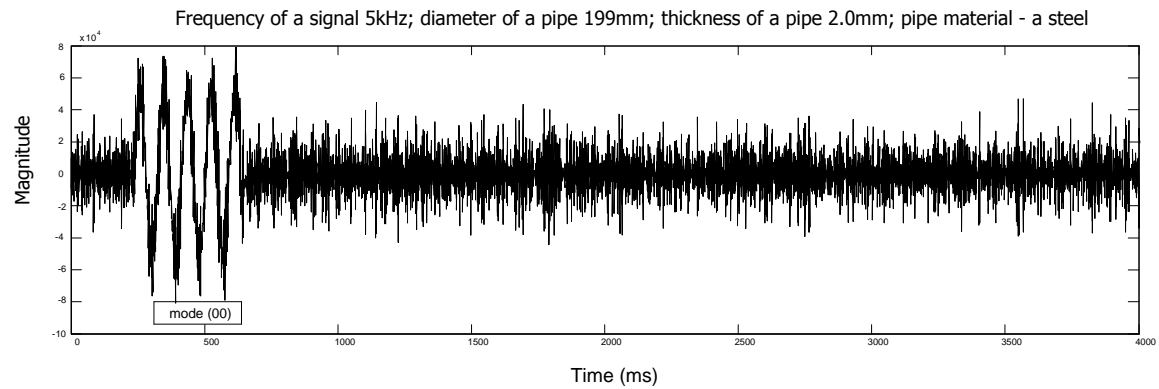

Fig. 3. Fields of extending of acoustical pressure 
The pulse with frequency $55 \mathrm{KHz}$ energises more amount of modes that leads to serious reverberation of a signal, thus, high-frequency modes damp more sweepingly, than low-frequency.

Thus, reflexions of an acoustical wave on an interface of the several medium having different density, will reduce energy of passed round acoustical pressure in a pipe and to increment the multiple-beam advance of waves. Therefore, in a cylindrical underground pipe the filled water, it is desirable to transmit information by means of a wave with a mode $(00)$ and to apply the filter to eduction of a mode (00) of the accepted pulses.

\section{References:}

[1] Zibrov, V.A., Sokolovskaya, O.V., Zibrova, N.M., Zanina, I.A, 2014. Remote ultrasound monitoring of underground water mains. Life Sci J, 11(10): 548-551.

[2] Misiunas, D., 2005. Monitoring and asset condition assessment in water supply systems, PhD thesis, Lund Univ., Lund.

[3] Dermile, R. and J. Saniie, 2001. Model-based estimation of ultrasonic echoes Part I: Analysis and algorithms. IEEE Transactions on Ultrasonics, Ferroelectrics and Frequency Control, 48(3): 787-802.

[4] Yang, C., Y. Liu and J. Yu, 2009. Prestressing Concrete Cylinder Pipe Monitoring Based on WSN. Journal of Information and Communication Technology, 2(2): 58-62.

[5] Jin, Y. and A. Eydgahi, 2008. Monitoring of distributed pipeline systems by wireless sensor networks. 2008 IAJC-IJME International Conference, International Association of Journals \& Conferences.

[6] O'Shea, P., 2000. Failure mechanisms for small diameter cast iron water pipes, PhD thesis, Southampton Univ., Southampton.

[7] Kokossalakis, G., 2006. Acoustic data communication system for in-pipe wireless sensor networks, PhD thesis, MIT, Massachusetts. 